\title{
THE INFLUENCE OF HYDROGEN ON MARTENSITIC TRANSFOMATION OF AUSTENITIC STEELS
}

\author{
A.I. Balitskii, L.M. Ivaskevich, V.M. Mochulskyi \\ Department of Hydrogen Resistance of Materials Karpenko Physiko-Mechanical Institute National Academy of \\ Sciences of Ukraine, 79601, Lviv, Ukraine
}

\begin{abstract}
The effect of hydrogen on the mechanical properties of the quenched and deformed specimens of $\mathrm{Cr}-\mathrm{Ni}$ and $\mathrm{Cr}-\mathrm{Mn}$ steels in $293 \ldots 773 \mathrm{~K}$ temperature range has been investigated. Non-hydrogenated and hydrogenated $(623 \mathrm{~K} ; 35$ $\mathrm{MPa} \mathrm{H}_{2} ; 5 \mathrm{~h}$ ) polished plane specimens with $3 \times 6 \times 20 \mathrm{~mm}$ working part were subjected to static tension with displacement rate of $0,1 \mathrm{~mm} / \mathrm{min}$ in helium and hydrogen under $35 \mathrm{MPa}$ pressure. It was established that all tested steels are sensitive to hydrogen degradation. The maximum effect of hydrogen has observed at room temperature, when decreased the elongation and reduction of area of specimens. Martensitic transformation of nitrogen-containing austenite (stable on air) was caused by hydrogen. In unstable steels hydrogen change the intensity of $\gamma-\alpha$ transformation. Formation of polygonal dislocation substructure by preliminary mechanical or thermomechanical treatment decreases the hydrogen degradation of steels.
\end{abstract}

The plastic and strength properties of unstable austenitic steels depend upon the intensity of the martensite transformation by deformation, which is controlled by alloying, mechanical working, and heat treatment $[1,2]$. Hydrogen has a significant influence on the processes of martensite formation and preliminary electrolytic or hightemperature hydrogen impregnation is accompanied both by a decrease and by the increase in the transformation temperature [3]. The role of deformation martensite in hydrogen embrittlement, especially in tests in a hydrogen medium, has been studied.

The purpose of this work is an investigation of the features of the deformation martensite transformation and hydrogen embrittlement of $12 \mathrm{Kh} 18 \mathrm{~N} 10 \mathrm{~T}, 06 \mathrm{Kh} 18 \mathrm{~N} 5 \mathrm{G} 9 \mathrm{AB}$ and $07 \mathrm{Kh} 13 \mathrm{G} 20 \mathrm{AN} 4$ steels under various conditions of hydrogen action.

Flat specimens with a cross section of $2 \times 6 \mathrm{~mm}$ hardened from $1273^{\circ} \mathrm{K}$ where short-term tensile tested with a rate of $10^{-4} \mathrm{sec}^{-1}$. A portion of the specimens was hydrogen-impregnated in advance in a working chamber with a hydrogen pressure of $35 \mathrm{MPa}$ and a temperature of $623^{\circ} \mathrm{K}$ for $5 \mathrm{~h}$. The temperature and time of impregnation were chosen so that the structure of the steels did not change during the hold. The original and hydrogenimpregnated specimens were tested both in hydrogen and in a neutral medium.

For the purpose of revealing the rule of phase transformations in hydrogen embrittlement and also as the result of the fact that work hardening is frequently used for hardening unstable steels, a portion of the experiments was made on specimens deformed in air in advance at room temperature at a rate of tension of $10^{-4} \mathrm{sec}^{-1}$.

The phase composition of the steels was determined with the use of a DRON-3 diffractometer in copper $\mathrm{K}_{\alpha-}$ radiation on $2 \times 4 \times 10 \mathrm{~mm}$ plates cut from the working portion of hardened or deformed specimens and also from the fracture zone of failed specimens. The integral intensities of the $(111)_{\gamma}$ and $(110)_{\alpha}$ lines were used for the quantitative calculations.

It was established (Figs. 1 and 2) that hydrogen has practically no influence on the short-term tensile strength in all conditions of the investigated materials. A significant reduction in the plasticity characteristics of the steels in the presence of hydrogen is observed at $293 \mathrm{~K}$. Then, with an increase in temperature, the influence of temperature is weakened and ceases at $573 \mathrm{~K}$ (Fig. 1). Here, as in the case of nickel heat-resistant alloys [4], the loss of strength of the material with an increase in temperature is accompanied by a marked decrease in the action of hydrogen.

In $12 \mathrm{Kh} 18 \mathrm{~N} 10 \mathrm{~T}$ steel the martensite transformation starts with a deformation of about $20 \%$ regardless of the test conditions (Fig. 3a). While in tension in air the martensite content in the specimens steadily increases, fracture in hydrogen occurs with a small quantity of this phase in the volume of the specimen. Suppression of the phase transformation was observed in tension in gaseous hydrogen of both preliminary hydrogen-impregnated and of nonhydrogen-impregnated specimens in which the a-phase content was 18 and $26 \%$, respectively, which is significantly less than after failure in air (Fig. 3). The difference in the plasticity characteristics of hydrogenimpregnated and non impregnated specimens broken in hydrogen is small (Fig. la). Therefore, there is a correlation between the change in the quantity of $\alpha$-martensite in the volume of the specimen and the degree of embrittlement.

aemail: balitski@ipm.lviv.ua

This is an Open Access article distributed under the terms of the Creative Commons Attribution-Noncommercial License (http://creativecommons.org/licenses/by-nc/3.0/), which permits unrestricted use, distribution, and reproduction in any noncommercial medium, provided the original work is properly cited. 

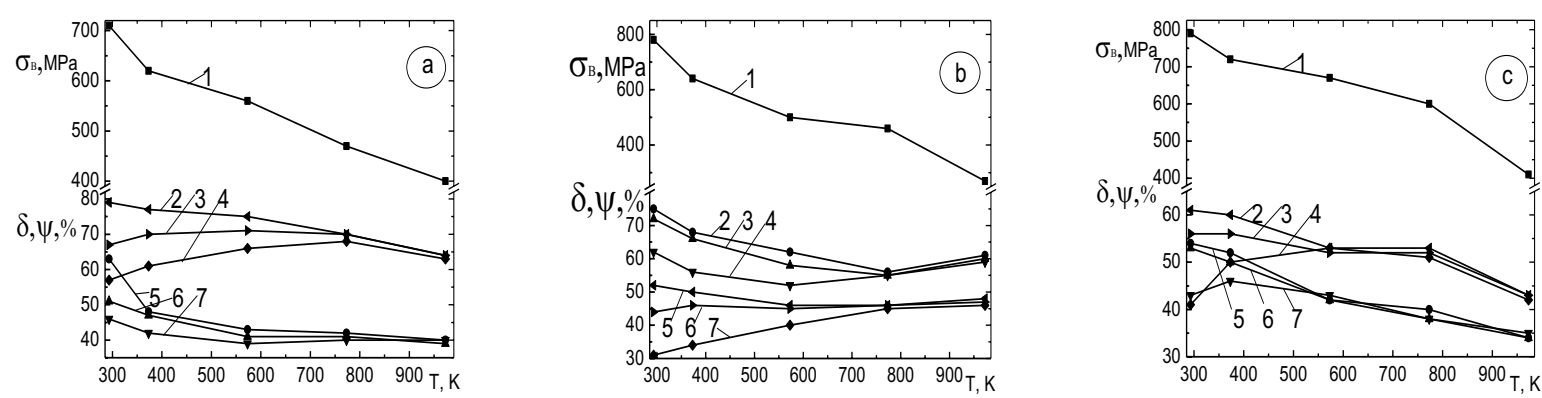

Fig. 1. Temperature dependence static tensile strength $\sigma_{\mathrm{u}}(1)$ and plasticity characteristics $\delta(2-4)$ and $\psi(5-7)$ of steel specimens $12 \mathrm{Khl}$ N10T (a), 06Khl8N5G9AB (b) and 07Kh13G20AN4 (c) in helium $(1,2,5)$, hydrogen at a pressure of $35 \mathrm{MPa}(3,4,6,7): 1-3,5,6$ - non-hydrogenetad specimens; 4,7 - hydrogenetad (623 K, $\left.35 \mathrm{MPa} \mathrm{H}_{2}, 5 \mathrm{~h}\right)$
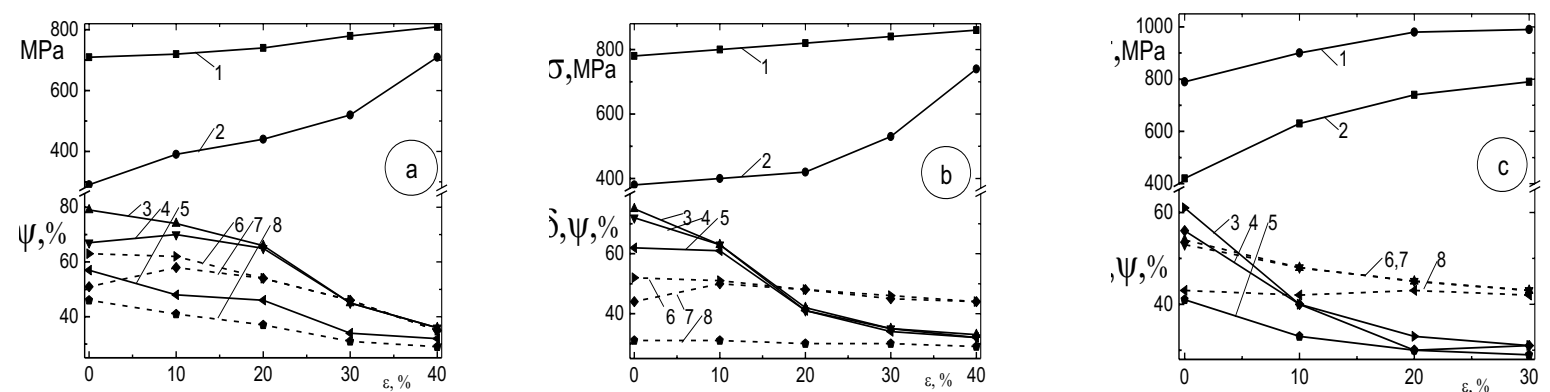

Fig. 2. Influence of the degree of deformation $\varepsilon$ on the characteristics of strength $\sigma_{\mathrm{B}}(1)$ and $\sigma_{0,2}(2)$ and plasticity $\delta(3-5)$ and $\psi(6-8)$ of $12 \mathrm{Khl} 8 \mathrm{~N} 10 \mathrm{~T}(\mathrm{a}), 06 \mathrm{Khl} 1 \mathrm{~N} 5 \mathrm{G} 9 \mathrm{AB}(\mathrm{b})$ and $07 \mathrm{Kh} 13 \mathrm{G} 20 \mathrm{AN} 4$ (c) steels at room temperature in air $(1,2,3,6)$; hydrogen at a pressure of $35 \mathrm{MPa}(4,7)$; hydrogen at a pressure of $35 \mathrm{MPa}$ after preliminary hydrogenation at $623^{\circ} \mathrm{K}$ and $35 \mathrm{MPa} \mathrm{H}_{2}$ for $5 \mathrm{~h}(5,8)$.

As in low-cycle fatigue tests [5], hydrogen embrittlement of $12 \mathrm{Kh} 18 \mathrm{~N} 10 \mathrm{~T}$ steel is caused by processes occurring on the surface of the specimen or at the crack tip. The comparatively small reduction in the plasticity of hydrogen-impregnated specimens tested in helium (Fig. la) may be explained by the fact that entry of hydrogen into the danger zone is controlled by diffusion from the volume of the specimen and occurs more slowly than adsorption from a gaseous medium. The reasons for embrittlement are the same in both cases and are related to the occurrence at points of high local stresses and hydrogen concentrations of martensite phase and to further failure in the interphase boundary $[6,7]$.

In $06 \mathrm{Kh} 18 \mathrm{~N} 5 \mathrm{G} 9 \mathrm{AB}$ steel, on the other hand, in the hydrogen-impregnated specimens the martensite transformation develops more intensely (Fig. 3) and, consequently, the relaxation possibilities are exhausted more rapidly $[1,2]$, Earlier it was established $[6,7]$ that the reason for the decrease in the stability of the austenite may be the reduction in stacking fault energy as the result of solution of hydrogen in the steel. Since steels alloyed with manganese and nitrogen already have low stacking fault energies, an additional reduction in it after hightemperature hydrogen impregnation leads to a significant change in intensity of the martensite transformation and, consequently, to a reduction in the plastic properties of the metal. Therefore there is practically no reduction in the plasticity of $06 \mathrm{Kh} 18 \mathrm{~N} 5 \mathrm{G} 9 \mathrm{AB}$ steel in an external hydrogen atmosphere but there is a marked decrease in it after preliminary hydrogen impregnation in tension both in hydrogen and in neutral media (Fig. Ib).

The features of hydrogen embrittlement of steels in the work-hardened condition are caused by the specifics of the martensite transformation. In testing deformed specimens of both materials in an atmosphere of hydrogen with an increase in the degree of plastic deformation, the influence of the hydrogen weakens and with deformations of more than $20 \%$ for $12 \mathrm{Khl} 18 \mathrm{~N} 10 \mathrm{~T}$ steel and $10 \%$ for $06 \mathrm{Khl} 18 \mathrm{~N} 5 \mathrm{G} 9 \mathrm{AB}$ steel the plasticity of the specimens broken in hydrogen and helium is the same (Fig. 2a, b). These values are close to the values of plastic deformation corresponding to the start of the martensite transformation of steels (Fig. 3). Consequently, the influence of external gaseous hydrogen is most significant at the start of deformation. With an increase in plastic deformation, that is, with more uniform distribution of it over the length of the specimen [8] and the formation of deformation martensite (Fig. 3) the action of the hydrogen atmosphere stops, which agrees well with known data [7] that the quantity of aphase formed in electrolytic hydrogen impregnation of type 18-8 steel decreases with an increase in the amount of preliminary cold tension.

High-temperature impregnation of deformed specimens leads to the accumulation of hydrogen at the boundary of the phases and a reduction in plasticity in further tension, which is characteristic of dual phase steels [9]. Therefore, embrittlement of hydrogen-impregnated specimens of $12 \mathrm{Kh} 18 \mathrm{~N} 10 \mathrm{~T}$ steel is markedly strengthened with an increase 


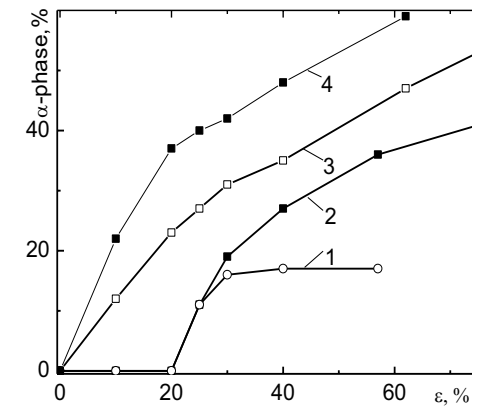

Fig. 3. Quantity of martensite phase in $12 \mathrm{Kh} 18 \mathrm{~N} 10 \mathrm{~T}(1,2)$ and $06 \mathrm{Khl} 18 \mathrm{~N} 5 \mathrm{G} 9 \mathrm{AB}(3,4)$ steels in relation to the degree of deformation in air $(2,3)$ and in hydrogen at a pressure of $35 \mathrm{MPa}$ after preliminary hydrogenation at $623^{\circ} \mathrm{K}$ and $35 \mathrm{MPa} \mathrm{H}_{2}$ for $5 \mathrm{~h}$ $(2,4)$

in the quantity of martensite from 0 to $25 \%$ in the range of preliminary deformations of $20-40 \%$ (Figs. $2 \mathrm{a}$ and $3 \mathrm{a}$ ). The plasticity of work-hardened hydrogen-impregnated specimens of 06Khl8N5G9AB steel drops, but to a lesser degree than of hardened (Fig. 2b). As is known [9], the hydrogen brittleness of dual-phase steels is strengthened only up to a certain content of the second phase, which for $06 \mathrm{Kh} 18 \mathrm{~N} 5 \mathrm{G} 9 \mathrm{AB}$ steel is reached with low values of plastic deformation. Since the influence of hydrogen on the properties of this steel is caused by intensification of the martensite transformation, with an increase in the degree of preliminary tension, when a significant quantity of aphase is formed before impregnation of the specimen with hydrogen, the sensitivity to hydrogen embrittlement decreases.

Consequently, hydrogen embrittlement of unstable austenitic steels is related to the change in intensity of formation of deformation martensite. After preliminary tension in air the sensitivity of 06Khl8N5G9AB steel to the action of hydrogen drops and in the work-hardened condition it possesses higher strength and plastic properties in the presence of hydrogen than $12 \mathrm{Khl} 8 \mathrm{~N} 10 \mathrm{~T}$ steel.

The established regularities of the effect of hydrogen on mechanical properties of chromium-manganese steels are attributable to the specific features of changes in their structure in hydrogen. Electron-microscopic and $x-$ ray analyses show that the energy of stacking faults significantly decreases owing to the hydrogenation of austenite steels $[1,5,8]$. It is known that the energy of stacking faults determines the stresses and, respectively, strains under which the transverse sliding of dislocations and the formation of a polygonal dislocation substructure occur [1,5,9]. These phenomena promote a uniform distribution of stresses and hydrogen in the metal, and, therefore, remove the danger of premature fracture. In stable austenite steels with a high energy of stacking faults, absorbed hydrogen cannot offer sufficient resistance to the formation of a polygonal substructure. In chromium-manganese steels alloyed by nitrogen, with a low energy of stacking faults, dissolved hydrogen hampers the transverse sliding of dislocations, which results in the formation of domains with increased local stresses and hydrogen concentration. Precisely this is probably the reason for the intensification of the martensite transformation, which is indicated by an increase in the content of a-phase in metastable steels $[1,2,5]$ and the formation of $\varepsilon$-martensite on fracture surfaces of specimens made of stable austenitic steel [2] and 07Kh13G20AN4 steels in this research. Apparently, there are similar reasons for the delayed fracture of hydrogenated specimens of $03 \mathrm{Khl} 6 \mathrm{~N} 12 \mathrm{G} 10 \mathrm{M} 5$ steel with 0.26 mass $\%$ of nitrogen [12], although it is stable and is considered to be insensitive to hydrogen embrittlement [13,14].

Table 1. Mechanical Properties of 07KM3G20AN4 Steel after Hardening and Tension at Increased Temperatures

\begin{tabular}{|c|c|c|c|c|c|}
\hline $\begin{array}{c}\text { Temperature of } \\
\text { deformation } T, \mathrm{~K}\end{array}$ & $\begin{array}{c}\text { Degree of } \\
\text { deformation } \varepsilon, \%\end{array}$ & $\sigma_{\mathrm{u}}, \mathrm{MPa}$ & $\sigma_{0,2}, \mathrm{MPa}$ & $\delta, \%$ & $\psi, \%$ \\
\hline \multirow{3}{*}{773} & 4 & $820 / 820$ & $440 / 440$ & $54 / 44$ & $50 / 49$ \\
\cline { 2 - 6 } & 15 & $860 / 860$ & $480 / 480$ & $54 / 53$ & $46 / 46$ \\
\cline { 2 - 6 } & 20 & $910 / 910$ & $520 / 520$ & $29 / 29$ & $32 / 30$ \\
\hline \multirow{3}{*}{973} & 5 & $810 / 810$ & $420 / 420$ & $53 / 52$ & $49 / 48$ \\
\cline { 2 - 6 } & 15 & $850 / 840$ & $460 / 460$ & $51 / 51$ & $46 / 46$ \\
\hline & 20 & $890 / 890$ & $490 / 490$ & $27 / 27$ & $28 / 27$ \\
\hline
\end{tabular}

Note: Figures in the numerators show the properties in air at $293 \mathrm{~K}$, and figures in the denominators indicate the properties of preliminarily hydrogenated $(35 \mathrm{MPa}, 623 \mathrm{~K}, 5 \mathrm{~h})$ specimens in hydrogen under a pressure of $35 \mathrm{MPa}$.

The effect of hydrogen on plastic deformation of chromium-manganese steels is a result of impediments to the transverse sliding of dislocations. For this reason, we approved methods of formation of the polygonal dislocation substructure before the hydrogenation of steels. One of these methods consists of plastic deformation (in a neutral environment) because, after attaining a certain level of strains, transverse sliding is facilitated in chromium-manganese austenite as well [5,15]. This fact explains the decrease in the sensitivity of plastic characteristics of steel to the action of hydrogen with increase in the preliminary tension (see Fig. 2). An additional effect can be related to the decrease in the length of the free path of dislocations in cold-worked specimens, which brakes hydrogen transportation by dislocations $[8,9,14]$.

Since cold deformation reduces the plasticity of steel, we formed the polygonal substructure by tension at elevated temperatures. We optimized the temperature-deformation regime according to the criterion of maximal strength and plasticity and the absence of microcracks. In view of the data of mechanical and electron-microscopic 
examinations, this can be attained by hardening from $1273 \mathrm{~K}$ and tension by $5-15 \%$ at $773-973 \mathrm{~K}$ (Table 1 ). If the temperature is less than $773 \mathrm{~K}$ and the deformation is less than $5 \%$, the polygonal substructure is not formed, and hydrogen endurance does not increase. A deformation by more than $15 \%$ is accompanied by the appearance of microcracks and a sharp fall of the plasticity not only in hydrogen but also in air. Tension at a temperature higher than $973 \mathrm{~K}$ causes a loss of strength of the steel.

Electron-microscopic examinations corroborated the appearance of the polygonal substructure (Fig. 4), i.e., the dislocation structure characteristic of stable chromium-nickel steels $[1,2,6,8]$ is formed as a result of hightemperature cold working under optimal conditions. Respectively, the resistance of steel to hydrogen degradation grew. Specimens treated under such conditions practically are not embrittled under simultaneous action of external and internal hydrogen. The strength of $03 \mathrm{Khl} 3 \mathrm{G} 20 \mathrm{AN} 4$ steel is comparatively low, and its reserve of plasticity is considerable.
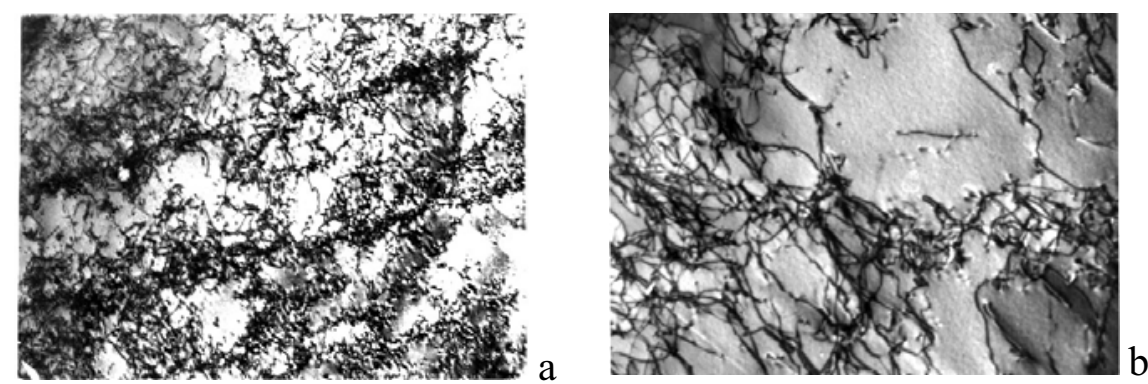

Fig. 4. Fine structure of 07Kh13G20AN4 steel after hardening (a) and deformation by $15 \%$ at $773 \mathrm{~K}(\mathrm{~b})$.

The high serviceability of chromium-manganese austenite with polygonized dislocation substructure in a hydrogen environment is corroborated by low-cycle fatigue tests, which are, as is known [7,16], one of the most sensitive criteria of hydrogen endurance of structural materials. We loaded the original specimens and those deformed by $5 \%$ at $973 \mathrm{~K}$ by sign-preserving pure bending with an amplitude of $1.6 \%$ and a deformation frequency of $0.5 \mathrm{~Hz}$ at room temperature in hydrogen under a pressure of $35 \mathrm{MPa}$. The ratio between the numbers of cycles up to fracture in air and hydrogen is 2200/1200 for the hardened specimens and 2900/2700 for the specimens after thermo-mechanical treatment. Preliminary hydrogenation of the latter according to the procedure described above results in an additional reduction of longevity to 2400 cycles.

Thus, the degree of hydrogen embrittlement of chromium-manganese austenite steels with solid-solution strengthening is determined by the dislocation substructure that is formed during deformation. Formation of the polygonal dislocation substructure by means of mechanical or thermomechanical treatment significantly decreases the sensitivity of steels to hydrogen degradation.

\section{References}

[1] T. Michler, J. Naumann.. International Journal of Hydrogen Energy 33, 2111 (2008).

[2] Shivanjuk V.N.,Foct J., Gavriljuk V.G. Scripta Mater. 49, 601(2003).

[3] L. S. Malinov and V. I. Konoton-Lyashko, Metalloved. Term. Obrab. Met.,. 1, 36 (1984).

[4] E. V. Savalei and I. N. Bogachev, Metalloved. Term. Obrab. Met 2, 18 (1984).

[5] V. I. Shapovalov, The Influence of Hydrogen on the Structure and Properties of Iron-Carbon Alloys [in Russian], Metallurgiya, Moscow (1982).

[6] G. G. Maksimovich, V. I. Kholodnyi, V. I. Belov, I. Yu. Tretyak, L. M. Ivas'kevich, and T. V. Slipchenko, Mater. Sci, 3, 53 (1984).

[7] A. N. Romaniv,., Mater. Sci,. 5, 33 (1981).

[8] M. Khasagava and M. Osava, Boshoku Gijutsu, Corros. Eng., 29, No. 9, 463-472 (1980)

[9] M. L. Holzworth and M. R. Loutan, Corrosion, 24, 4, 110 (1968).

[10]I. A. Shapovalov, V. Ya. Ul'ichev, and N. N. Antonova, Probl. Prochn., 3, 91(1983).

[11]R. G. Davies, Scripta Met., 17, 889(1983).

[12] S. O. Suvorova and G. A. Filippov, Metalloved. Term. Obrab. Met., 3, 24 (1986).

[13] Yu. I. Archakov, Hydrogen Corrosion of Steel [in Russian], Metallurgiya, Moscow (1985).

[14]B. A. Kolachev, Hydrogen Embrittlement of Metals [in Russian], Metallurgiya, Moscow (1985

[15] G. N. Grikurov and F. N. Tavadze, Izv. Akad. Nauk SSSR, Met., 5, 211 (1977).

[16] V. I. Tkachov, V. I. Kholodnyi, and I. R. Levina, Serviceability of Steels and Alloys in a Hydrogen Environment, Vertikal', L'viv (1999). 